مراقبة فيضان نهر دجلة وتأثيره على المناطق السكنية والسياحية في مدينة الموصل Sentinel-2 باستخدام المرئيات الفضائية للقمر الاصطناعي

\author{
ايمن طلال الصائغ \\ عبدالرحمن رمزي قبع \\ مركز التحسس النائي \\ جامعة الموصل \\ (تاريخ الاستلام 2020/1/20 تاريخ القبول 2020/5/2)
}

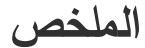

يعد العراق من الدول ذات الوفرة المائية لما يحتويه من مصادر مائية تتوزع بين دائمية وموسمية الجريان،

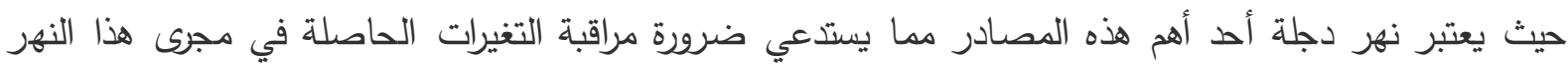
ودراسة المتغيرات البيئية والمناخية والتعروية التي تعمل على تغيير الخصائص المورفودايناميكية لهذا المورد،

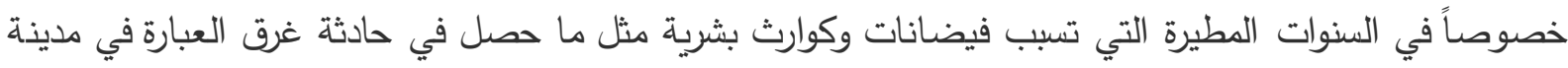

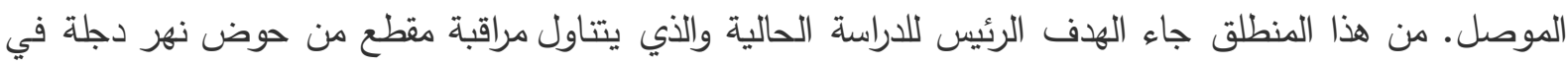
بداية دخوله الى مدينة الموصل ودراسة تأثيرات تغير منسوب المياه خلال المواسم المختلفة على أكتاف النهر

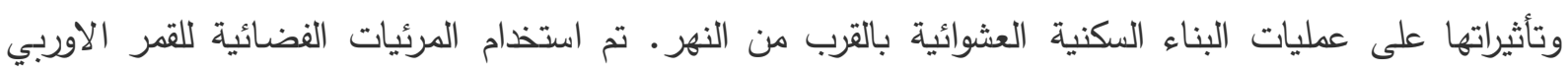
الجديد Sentinel ذو دقة التمييز الدكانية العالية والتي تصل الى 10 م لكل بكسل، والذي كان آخر إطلاق لسلسلته

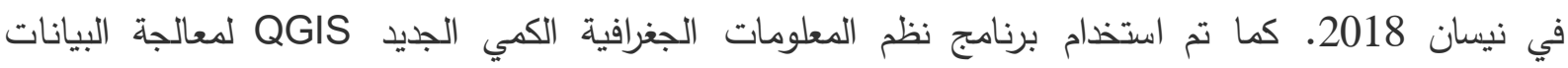
الفضائية واستخدام وسائل المعالجة الرقمية لاستخلاص المعلومات ومراقبة التغيرات في مقطع من النهر . إذ تم

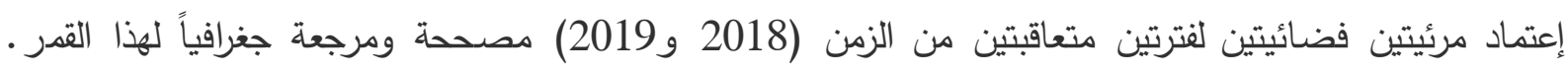

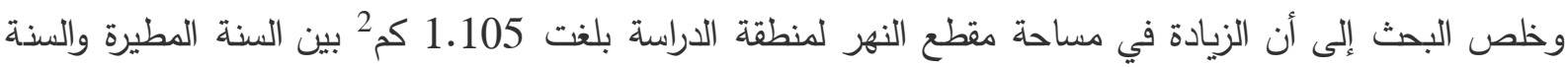
القليلة الأمطار أي بزيادة تقريبية تتجاوز 50\%. لذا يوصي الباحثون بندع تثيييد أي مناطق سكنية أو مناطق

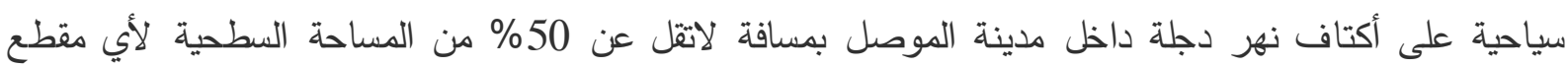
لللنهر داخل الدينة واضافتها للمناطق الدحرمة. الكلمات الدالة: القمر الاصطناعي Sentinel؛ برنامج QGIS، فيضان نهر دجلة، التحس النائي.

\section{Detecting the Tigris River Flood and its Impact on Residential and Touristic Areas in Mosul City Using Sentinel-2 Satellite Images}

\author{
Abdalrahman R. Qubaa \\ Ayman T. Alsayiegh
}

Remote Sensing Center

University of Mosul 


\section{ABSTRACT}

Iraq is one of the countries with water abund ance because it contains water sources distributed between permanent and seasonal flow. The Tigris River is considered as one of these the most important sources, which requires the necessity to monitor the changes that had been taken place in the course of this river and to study the environmental, climatic and erosional changes that were responsible to change the morphodynamic properties of this resource, especially in the rainy years that cause floods and human disasters, such as what happened in the accident of the sinking of the ferry in the city of Mosul. Consequently, the main objective of the current study is based on observing a selected section of the Tigris River basin at the beginning of its entry into Mosul city and studying the effects of changing the water level during different seasons on the banks of the river and its effects on random housing construction near the river. A new European satellite (Sentinel) is used which has high spatial resolution of up to $10 \mathrm{~m}$ per pixel, which was the last launch of its series in April 2018. The new QGIS program is also used to process the satellite data, using digital processing methods for interpretation, and to monitor changes in the studied section of the river. Two satellite images are adopted for two consecutive periods (2018 and 2019), corrected and geographically referenced.

The conclusion is that there is an increase of $1.105 \mathrm{~km} 2$ in the area of the studied river section between the rainy year and the less rainy year, i.e. an approximate increase in excess of $50 \%$. Therefore, the researchers recommend preventing the construction of any residential or touristic projects on the both Tigris River banks within at city of Mosul, and add to the forbidden areas a distance of not less than $50 \%$ of the surface area of any section of the river inside the city.

Keywords: Sentinel Satellite, QGIS Program, Tigris river flood, Remote Sensing.

\section{المقدمة}

يوفر التحس النائي وسيلة أكثر دقة في مراقبة التغيرات الحاصلة في الظواهر الطبيعية الديناميكية، وإن أحواض الأنهار هي من أكثر هذه الظواهر أهمية لارتباطها الكبير في نمو وتقدم وازدهار المدن الواقعة على هي ضفافها (Zhang et al., 2008). يشكل نهر دجلة العصب الرئيس للحياة في مدينة الموصل مركز محافظة نينوى، اضافة الى مدن اخرى في العراق مثل صلاح الدين، والتي اشتهرت بالزراعة وعرفت بسلة خبز العراق، ويعتبر هو النهر الرئيس الذي يقسم المدينة الى جانبين ويعتمد عليه لأغراض الشرب والري والاستخدامات الصناعية. بينت الدراسات والإحصائيات الأخيرة بشأن نهر دجلة فقدانه الكثير من المياه خلال السنوات الأخيرة نتيجة للمتغيرات المناخية وبناء السدود التي أقيمت في تركيا على مجرى النهر وأخذ مجرى النهر بالتغاير وفقاً لهذه التغيرات (خطاب ومصطفى، 2009)، كل ذلك أدى إلى زحف المناطق السكنية وعمليات البناء العشوائية على الاراضي الزراعية

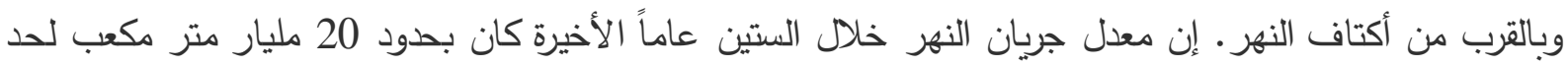
عام 1999، إلى أن وصلت إلى تسعة مليارات متر مكعب تقريباً خلال العقدين الماضيين (مقاييس سجلات نهر دجلة، 2006)، لهذا مرت ظروف جفاف للنهر نتيجة المتغيرات المناخية وسياسة الدول التي تثارك العراق نهرياً في السنوات السابقة، في حين ان وفرة الامطار لسنة 2019 الاستثنائية رفعت مخزون المياه إلى مرتين ونصف 
ضعف السنوات السابقة (وزارة الموارد العراقية، 2019). ان فترة شحة الوفرة المائية للنهر أدت إلى ظهور مساحات جافة شاسعة على أكتاف النهر وإلى استيلاء الجمعيات التعاونية السكنية على هذه المساحات وبيعها للمواطنين كأراضي سكنية. في حين ان كثرة الامطار التي حدثت خلال سنة 2019 ادت الى زيادة مناسيب المياه في النهر بشكل كبير اضافةً إلى فتح بوابات سد الموصل لتصريف المياه الوفيرة القادمة من مصادر النهر الرئيسية مما أدى لئه ذلك إلى غمر مساحات كبيرة من أكتاف النهر بالمياه والتي كانت قد تحولت إلى مناطق شبه سكنية. يهدف هذا البحث لتحقيق هدفين اساسيين هما: الاول، ايقاف تزايد الزحف السكاني الكبير على الاراضي الزراعية في منطقة كتف النهر ومنع عمليات البناء العشوائي الخاطئة في محرمات النهر والتي تتذر بغرق هذه الاراضي في مواسم الامطار اضافة الى توجيه الانظار الى المخاطر التي تواجه المناطق السياحية المتواجدة على ضفاف النهر في موسم زيادة الامطار مما قد ينتج عن ذلك حالات فيضان وخسائر وكوارث بشرية ومادية، كما حثث في حادثة العبارة الاليمة في جزيرة الموصل السياحية سنة 2019. والهدف الثاني، بيان امكانيات التقنيات الحديثة سواء كانت الاقمار الاصطناعية او برامجيات نظم المعلومات الجغرافية المستخمة في هذا البحث لدرء مخاطر الكوارث البشرية

تuantum Geographical Information تم استخدام برنامج نظم المعلومات الجغرافية الجديد Sentinel لمعالجة البيانات الفضائية للقمر الاصطناعي الاوربي QGIS والذي يرمز له اختصارا System والملتقطة لمدينة الموصل بغية تحقيق اهداف هذا البحث.

\section{Sentinel القمر الاصطناعي}

القر الاصطناعي Sentinel هو قمر أوروبي خصص للتصوير الضوئي للأرض، حيث وفر هذا القدر فرصة رائعة لمراقبة الغطاء النباتي والارضي بسبب خصائصه المكانية والطيفية والزمانية المحسنة مقارنة مع هع الاقمار الامريكية لاندسات والفرنسية SPOT SPrampton et.al. 2013). Sentinel2) هو القهر الثاني من جيل Sentinel الذي أطلقته وكالة الفضاء الأوروبية في عام 2017 كجُزء من البرنامج الاوربي كوبرنيكوس الفضائي. يعتمد نظام Sentinel2 على العمليات المتزامنة لاثثين من الأقمار الاصطناعية المتماثلة التي تحلق على مدار واحد ولكن بطور 180 درجة، وبهذا يتمكن المشروع من مسح الارض كل خمسة ايام. يتميز هذا القدر

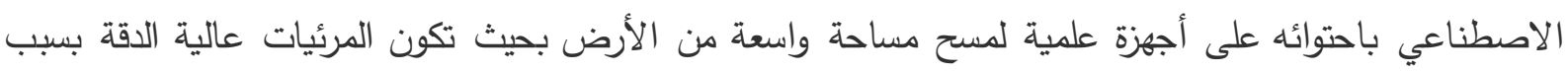
التصوير متعدد الأطياف الذي احتوى على 13 حزمة طيفيَّة موضحة في الجدول (1) وبدقة تمييز تصل الى 10 م لكل بكسل. والهدف هو مسح الأرض من عدة جهات وتصوير كل مكان في الأرض لمعرفة التغييرات الجغرافية المناخية والطبيعية وكما موضح في الثكل (1)، مع تغطية لكل بيان بطول 290 كم. ان الدقة الزمانية العالية لمجموع القدرين مترافقة مع سرعة توصيل النتائج لها، حيث يتم توصيل بيانات القمر خلال 24 ساعة للاستخدام العادي وتصل الى 10 دقائق في حالات الطوارئ، اضافة الى توفيره نطاقات طيفية اكثر من القر الامريكي

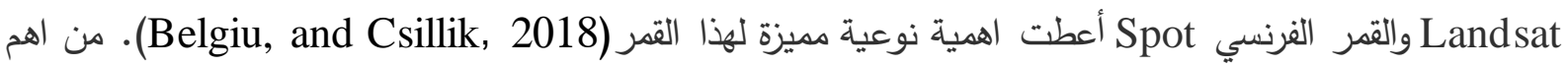
التطبيقات الرئيسة للقدر هي مراقبة المياه الساطية والداخلية، التنبؤ بالمخاطر ورسم خرائط الكوارث، الزراعة 
والغابات، وتغيير استخدام الأراضي، وتغير غطاء الأرض، رسم خرائط للمتغيرات الجيوفيزيائية مثل محتوى الكلوروفيل الورقي، محتوى الماء الورقي (Gatti, and Galoppo, 2018).

الجدول1: دقة التمييز المكانية، مركز الطول الموجي، العرض الترددي للباند لمرئيات القمر Sentine12.

\begin{tabular}{|c|c|c|c|c|c|}
\hline $\begin{array}{c}\text { الترددي للباند الحزمة } \\
\text { (nm) } \\
\text { (nm) }\end{array}$ & $\begin{array}{c}\text { مركز الطوجي للباند } \\
\text { (nm) } \\
\end{array}$ & دقة التمييز & فائدة الباند بالنسبة للطيف & اسم الباند & ت ت \\
\hline 20 & 443 & 60 & در اسة البحار & $\mathrm{B} 1$ & .1 \\
\hline 65 & 490 & 10 & الازرق & $\overline{\mathrm{B} 2}$ & .2 \\
\hline$\overline{35}$ & 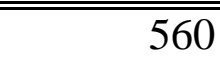 & 10 & |الاخضر & $\overline{\mathrm{B} 3}$ & 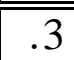 \\
\hline$\overline{30}$ & "665 & 10 & الاحمر & $\overline{\mathrm{B} 4}$ & 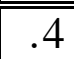 \\
\hline 15 & $\overline{7705}$ & 20 & دراسة النباتات & $\overline{\mathrm{B} 5}$ & .5 \\
\hline 15 & 740 & 20 & در اسة النباتات & B6 & .6 \\
\hline 20 & 783 & 20 & در اسة النباتات & B7 & .7 \\
\hline$\overline{1115}$ & 842 & 10 & تحت الحمراء القريبة & $\overline{\mathrm{B} 8}$ & .8 \\
\hline 20 & 865 & 20 & در اسة النباتات & $\overline{\mathrm{B} 8 \mathrm{~A}}$ & .9 \\
\hline 20 & 945 & 60 & بخار الماء & B9 & .10 \\
\hline 30 & 1375 & 60 & تحت الحمر اء القصيرة & B10 & .11 \\
\hline$\overline{90}$ & 1610 & 20 & تصت الحمر اء القصبرة & B11 & .12 \\
\hline 180 & 2190 & 20 & تصت الحمر اء القصيرة & B 12 & .13 \\
\hline
\end{tabular}

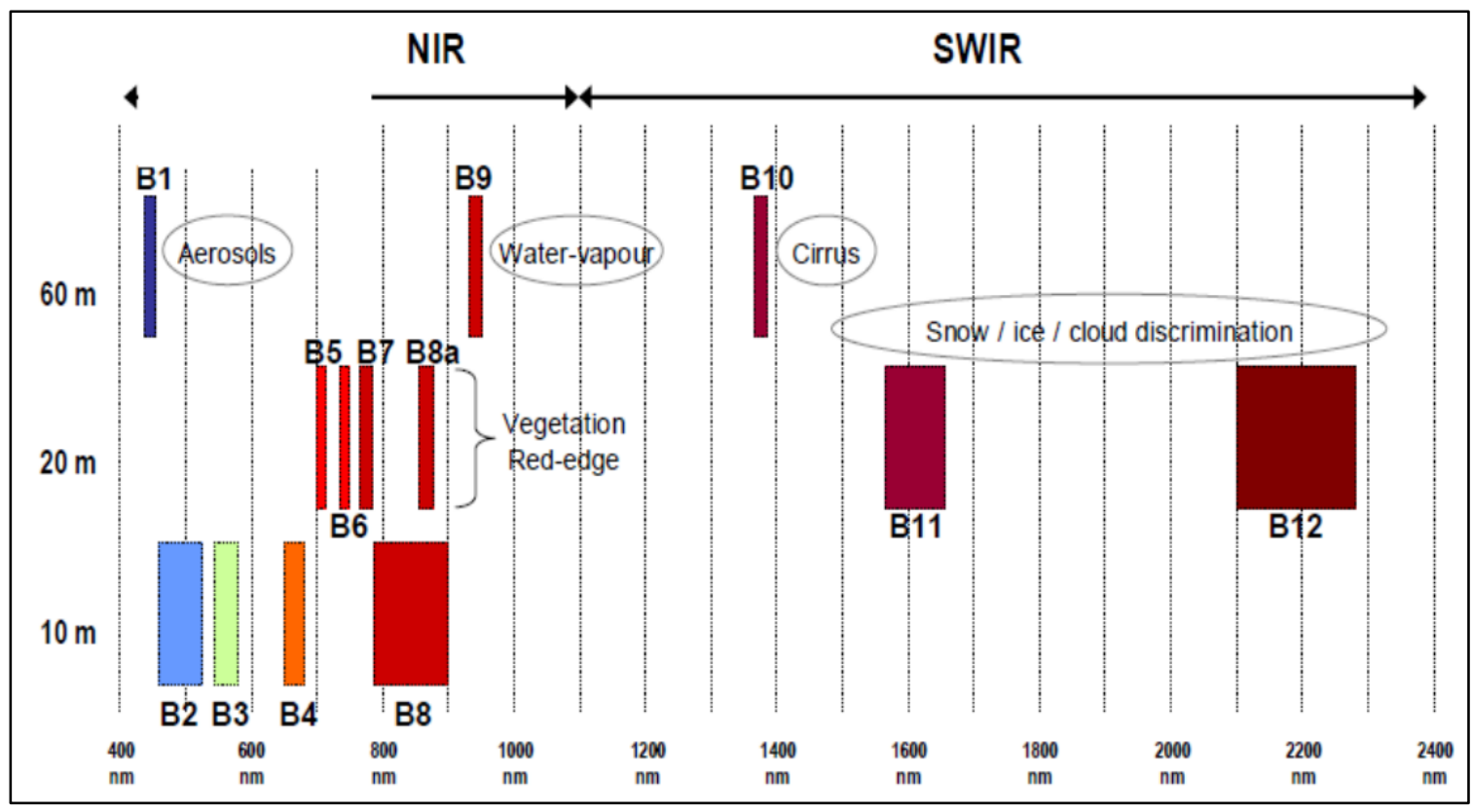

Gatti, ) Sentine12 الثكل 1: المتحسات متعددة الاطياف مقابل دقة التمييز المكانية للمتحسات التي يحملها القمر .(and Galoppo, 2018 
تم استخام مرئيتين فضائيتين في هذا البحث للقمر Sentinel2 ملتقطة في سنتين مختلفتين (20182019) ولنفس المنطقة واستخدامهما للمقارنة لإظهار زيادة منسوب مياه نهر دجلة وتأثيره على أكتاف النهر كما سيتم تفصيل ذلك في فقرات لاققة.

\section{Qرنامج نظم المطومات الجغرافية Quantum GIS}

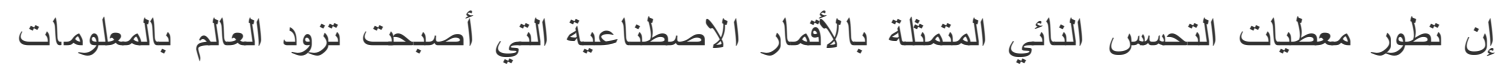

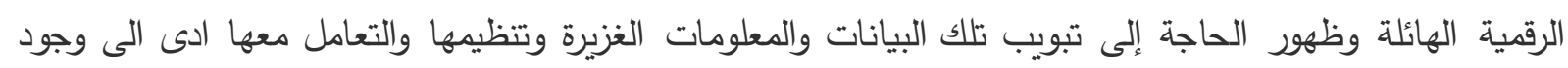

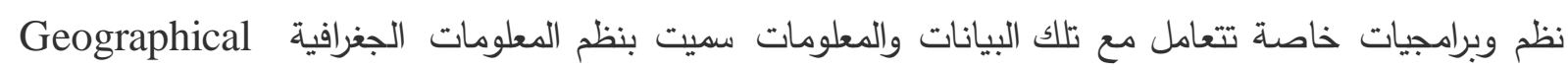
Information System GIS طريقة واسلوب علمي لتتظيم المعلومات والبيانات الجغرافية بوساطة الحاسوب وربطها بمواقعها الجغرافية اعتمادا

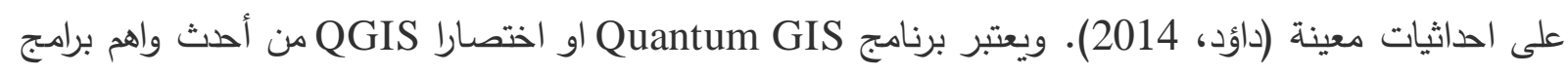

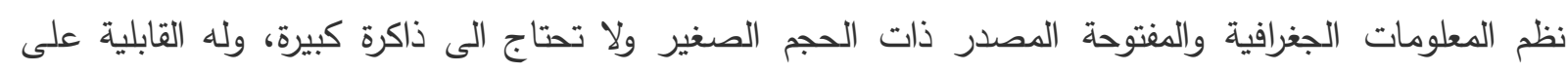

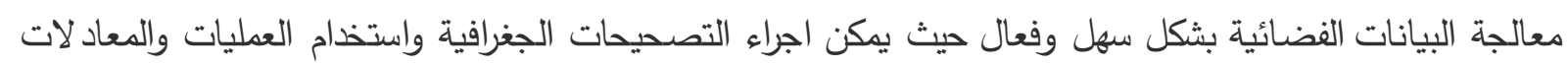
الرياضية لاستنباط الاستتاجات التحليلية للظواهر الجغرافية المختلفة (شعبان، 2017). تم استخدام نسخة

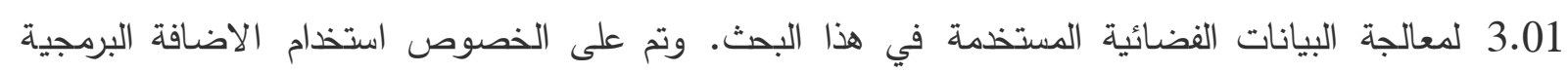
الجديدة المطورة في البرنامج المسماة التصنيف شبه الاوتوماتيكي (Pemi-Automatic Classification (Plugin

\section{موقع منطقة الدراسة}

تم اختيار منطقة الدراسة عند بداية دخول النهر من الجهة الثمالية لددينة الموصل، لأهمية المنطقة لكونها منطقة قرببة من الاحياء السكنية والتي تقع بين منطقة حي العربي وحي الرشيدية والمتعارف على تسميتها بمنطقة

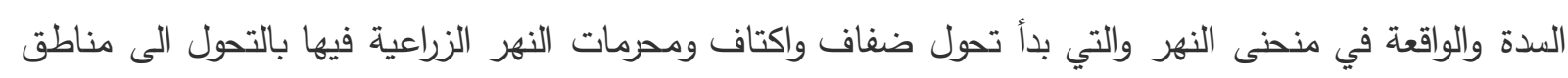

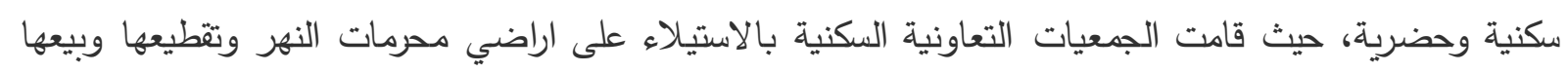

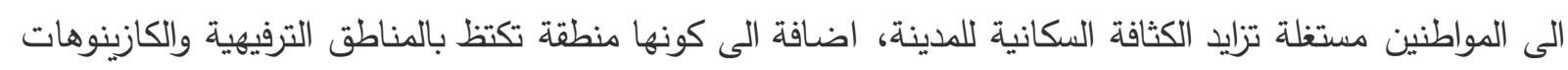

السياحية على ضفاف النهر وكما موضح في الثكل (2).

\section{تهيئة المطيات}

تعتمد تطبيقات التحس النائي على الصور الجوية والمرئيات الفضائية، والتي تعد من أهم مصادر

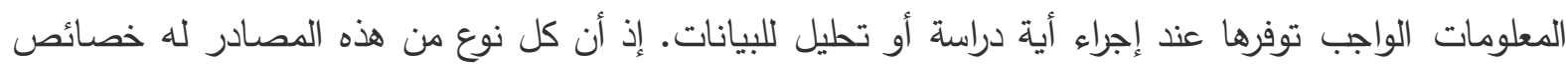

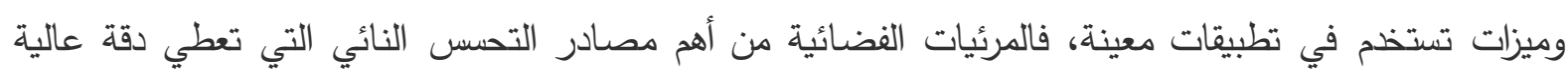

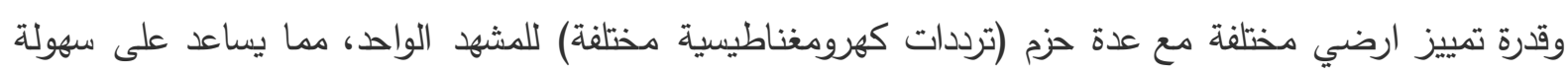


تحليل البيانات الصورية وتقفيرها والحصول على المعلومات اللازمة لإجراء الدراسات في مختلف المجالات سواء الجغرافية او تطبيقات التحس النائي (Lillesand, et al., 2008).

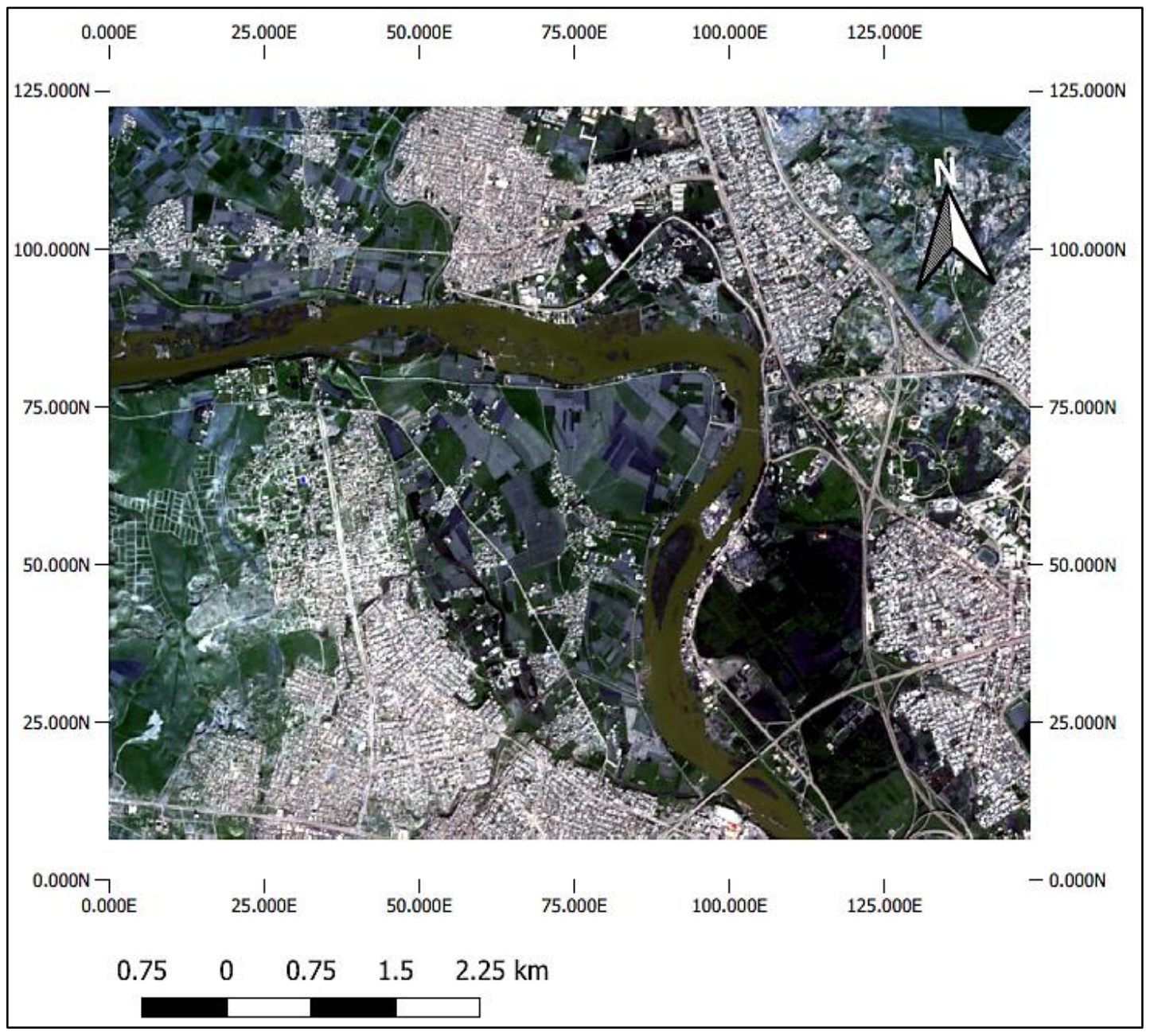

الثكل 2: موقع منطقة الدراسة، مقطع نهر دجلة عند المدخل الثمالي لمدينة الموصل.

تم استخدام مرئيتين فضائيتين لسنتين متعاقبتين بالزمن (2018 و2019) مصححة ومرجعة جغرافيا للقدر الاصطناعي Sentinel2. حيث تم تحميل مرئيتين فضائيتين للقرر عن طريق المنصة الاوربية الرسمية المسماة (https://scihub.copernicus.eu/) (Copernicus Open Access Hub) تحميل بيانات الأقمار الاصطناعية الثلاثة (Sentinel 1,2 and 3).

\section{منهجية العمل}

يعتمد التوصل إلى نتائج دقيقة في الدراسات التطبيقية للمعالجة الرقمية بصورة أساسية على مراحل هذه

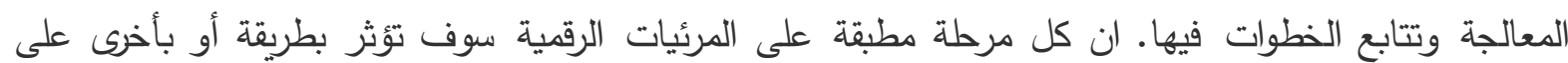
صفات المرئية الرقمية والتي بدورها تتحكم بقيمة ونتائج القياسات المستنبطة منها (Jensen, 2005). 
لكي تكون المقارنة علدية ودقيقة، تم تحميل مرئيتين لنفس المنطقة: الاولى لفصل الثتاء في الثهر الثاني لسنة 2018 والثانية في نفس الثهر ولكن لسنة 2019. تم الاعتماد في الدراسة على خطوات العمل الموضحة في الثكل (3) مع ملاحظة ان جميع الخطوات تم تطبيقها على المرئيتين للسنتين 2018 و2019 ولكن تم توضيح الخطوات العملية في الاشكال اللاحقة كمثال توضيحي للمرئية الملتقطة لسنة 2019 فقط.

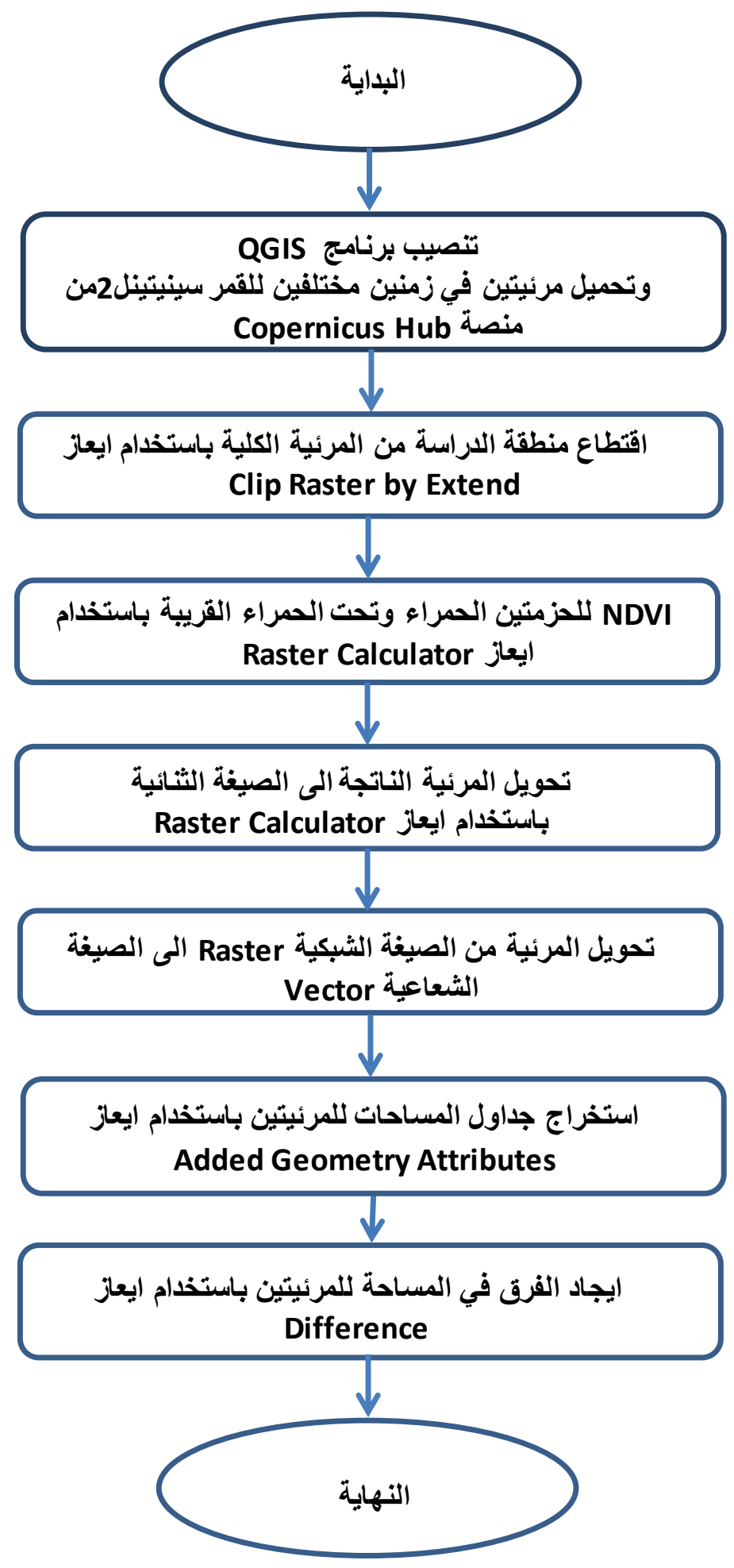

الثكل 3: مخطط يوضح منهجية وخطوات العمل المعتمدة في البحث. 
بعد تحميل البيانات من موقع الوكالة الاوربية وتحميل برنامج QGIS من موقعه الرسمي، تم اختيار الحزم الطيفية الثلاثة (432) كونها تغطي المجالات الترددية التي تقع ضمن الاطياف المرئية لعمل تركيب لوني بالألوان الطبيعية للتحقق من موقع الابنية السكنية المستحثثة، بحيث ان القناة الحمراء الرابعة تأخذ التسلسل 1 والقناة الخضراء الثالثة تأخذ التسلسل 2 والقناة الثانية الزرقاء تأخذ التسلسل 3 وبما ان هذه الحزم تمتلك دقة مكانية عالية (10 متر ) فسوف تتتج مرئية طيفية ملونة RGB ذات دقة (10 متر ) ايضا كما مبين في الشكل (4)، حيث يمثل الثكل 4-أ المرئية الملونة المركبة الملتطة للمنطقة بتاريخ 2018/2/1، والثكل 4-ب المرئية الملونة الملتطة 2019/2/21 20تاريخ
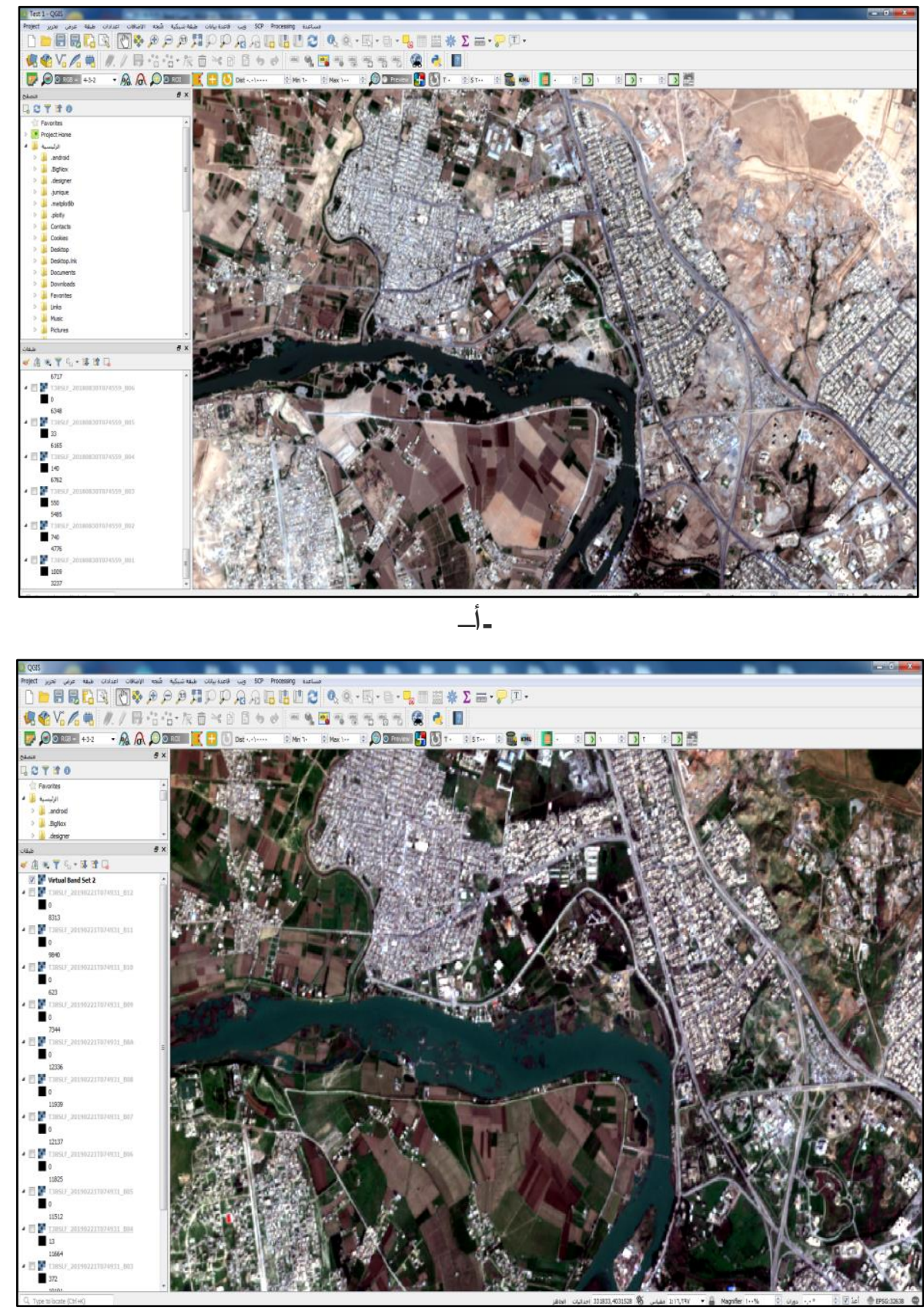

-ب

الثكل 4: عمل تركيب المرئية الملونة بالألوان الطبيعية لمنطقة الدراسة في موسمين مختلفين باستخدام برنامج QGIS. 


$$
\begin{aligned}
& \text { عبدالرحمن رمزي قبع و ايمن طلال الصائغ } \\
& \text { (-أ- تمثل المرئية بتاريخ 2018، -ب- تمثل المرئية بتاريخ 2019). }
\end{aligned}
$$

الخطوة الثانية هي استقطاع منطقة الدراسة وذلك لتصغير حجم المرئية التي نتعامل معها توفيرا للجهد والوقت الازم. وتم ذلك باستخدام الايعاز Clip Raster by Extend والموجود في قائمة الطبقة الشبكية وكما في الثكل

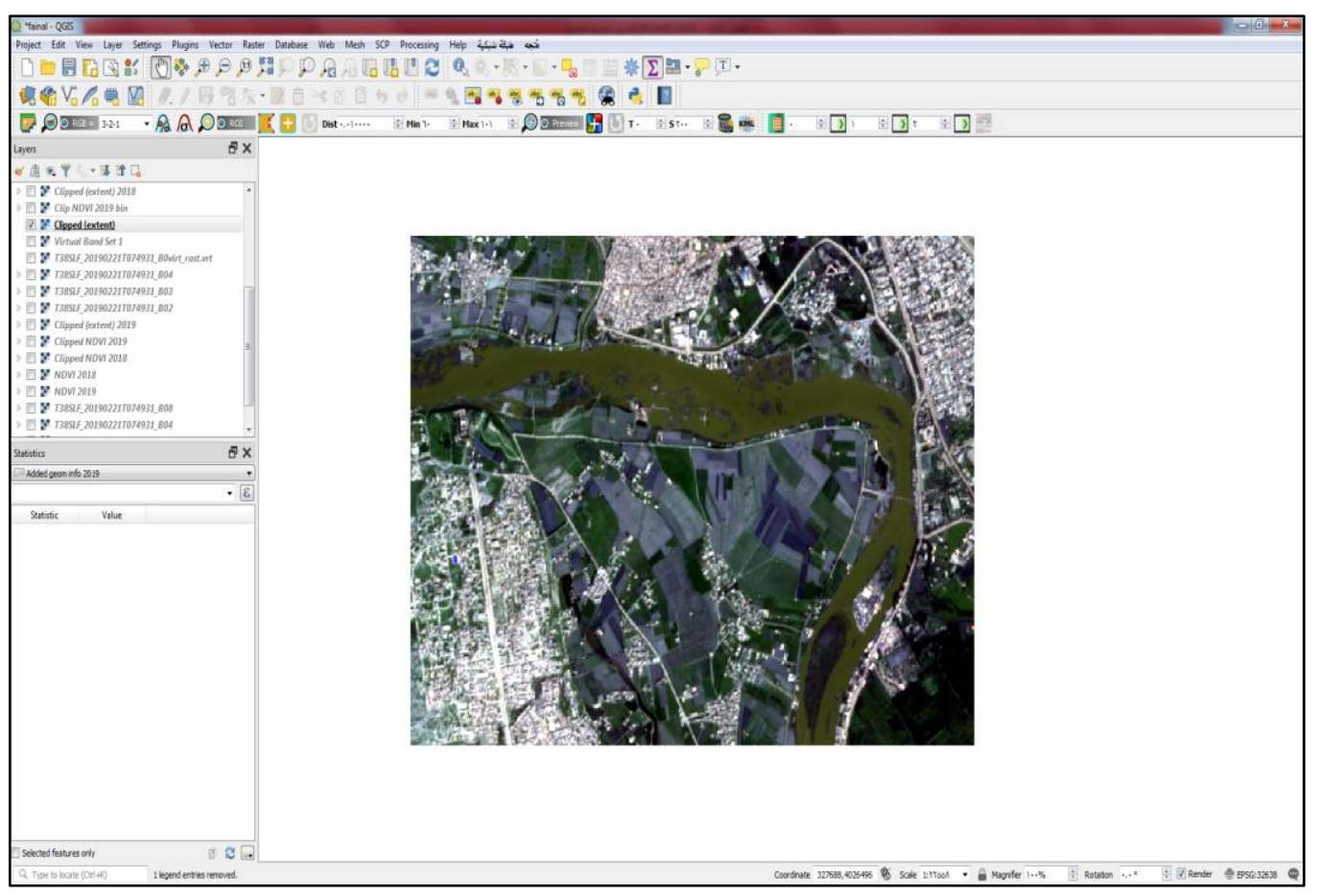

الثكل 5: اقتطاع منطقة الدراسة للمرئية الفضائية لسنة 2019.

الخطوة الثالثة هي استخراج وفرز المياه عن بقية الاصناف الارضية بالاستفادة من استخدام معادلة معامل الخضرة عن طريق المعادلة (1) حيث تم استخدام الايعاز Raster Calculator والموجود في قائمة Raster: $N D V I=(N I R-B A N D$ red $) /(N I R+B A N D$ red $)$

اذ تم استخدام الحزمة الحمراء مع الحزمة تصت الحمراء القريبة في المعادلة، والقيم الناتجة تكون محصورة بين [-1+1] وبإدخال قيمة حدية نستطيع تحيد قيم الماء حيث تكون قيمة الماء سالبة دائما وكما موضح في الثكل (6). الخطوة الرابعة هي انتاج مرئية ذات صيغة ثنائية، بحيث تخصص قيمة (0) للمناطق غير المغمورة بالمياه وقيمة (1) للمناطق التي تغطيها المياه حتى نستطيع تحويل البيانات إلى الصيغة الشعاعية بشكل أسرع وعن طريق استخدام اداة الحاسبة ايضا Raster Calculator وباستخدام المعادلة (2) وكما موضح بالثكل (7): 


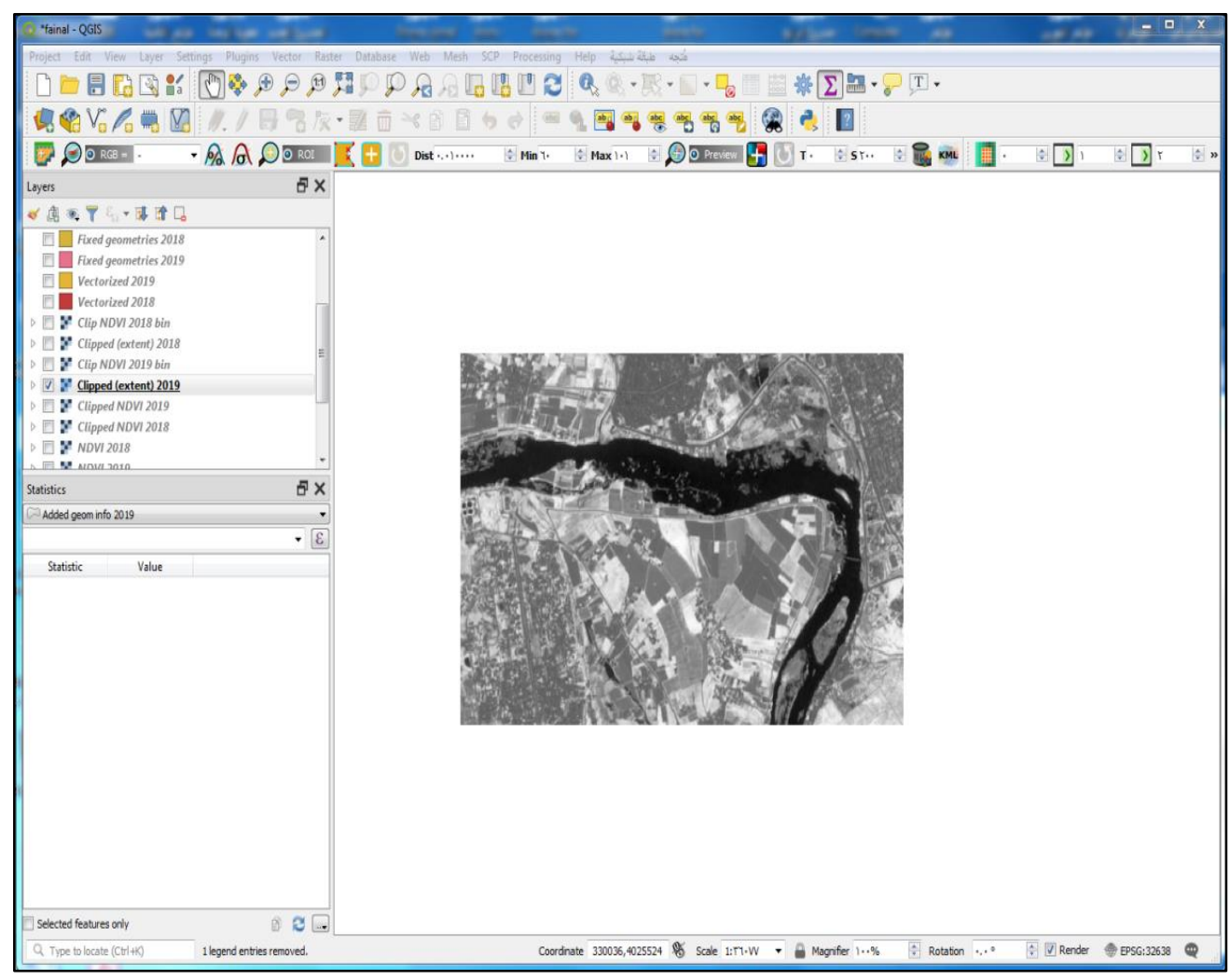

الثكل 6: عمل تصنيف لاستخراج وفرز المياه عن بقية الاصناف الارضية.

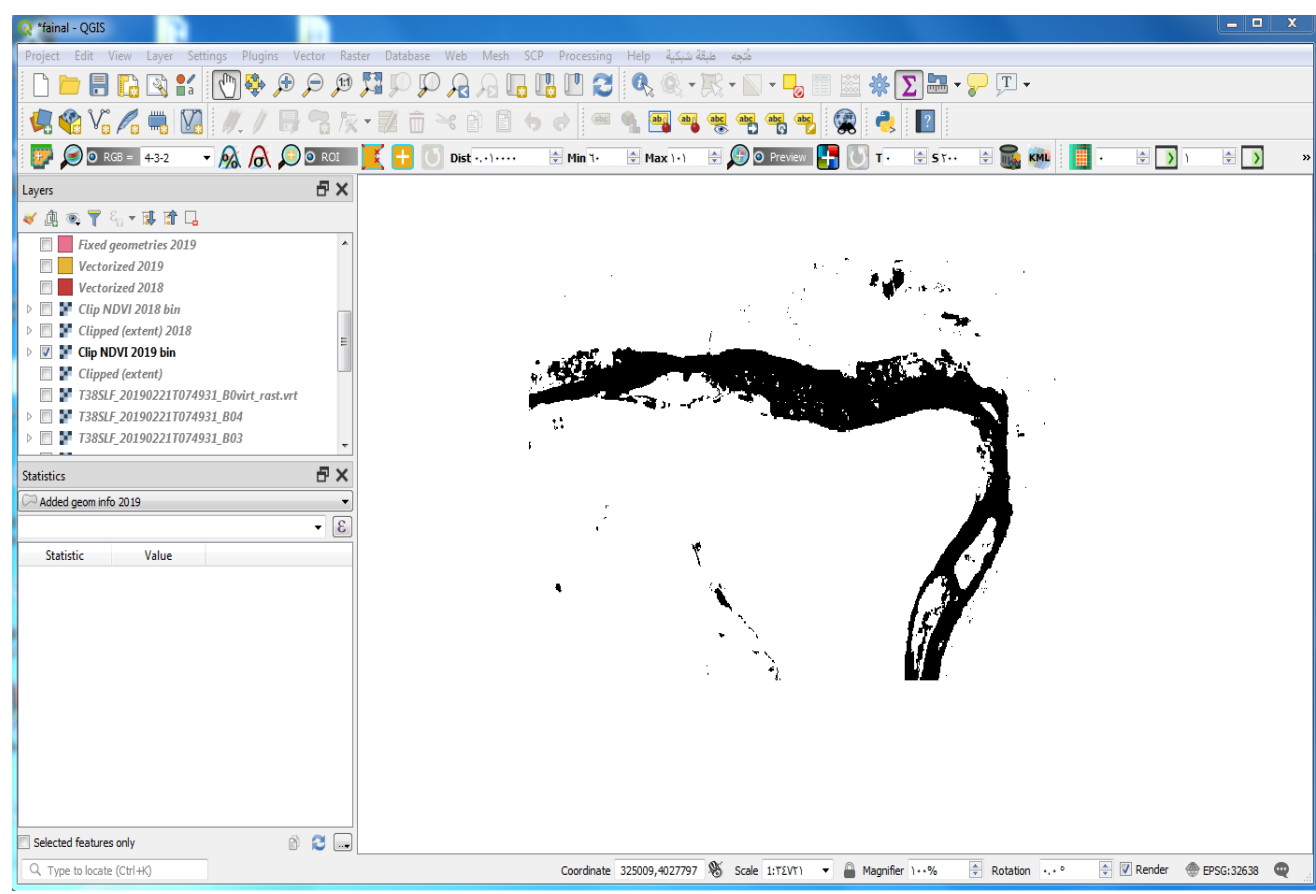

الثكل 7: تحويل المرئية الى مرئية ثنائية تمهيدا لتحويلها الى الصيغة الثعاعية.

الخطوة الخامسة هي تحويل البيانات من الصيغة الثبكية إلى الصيغة الثعاعية لكي نتمكن من حساب

Polygonize المساحات ويتم ذلك عن طريق الايعاز الموجود في قائمة طبقة شبكية في شريط القوائم وهو

(Raster to Vector) 


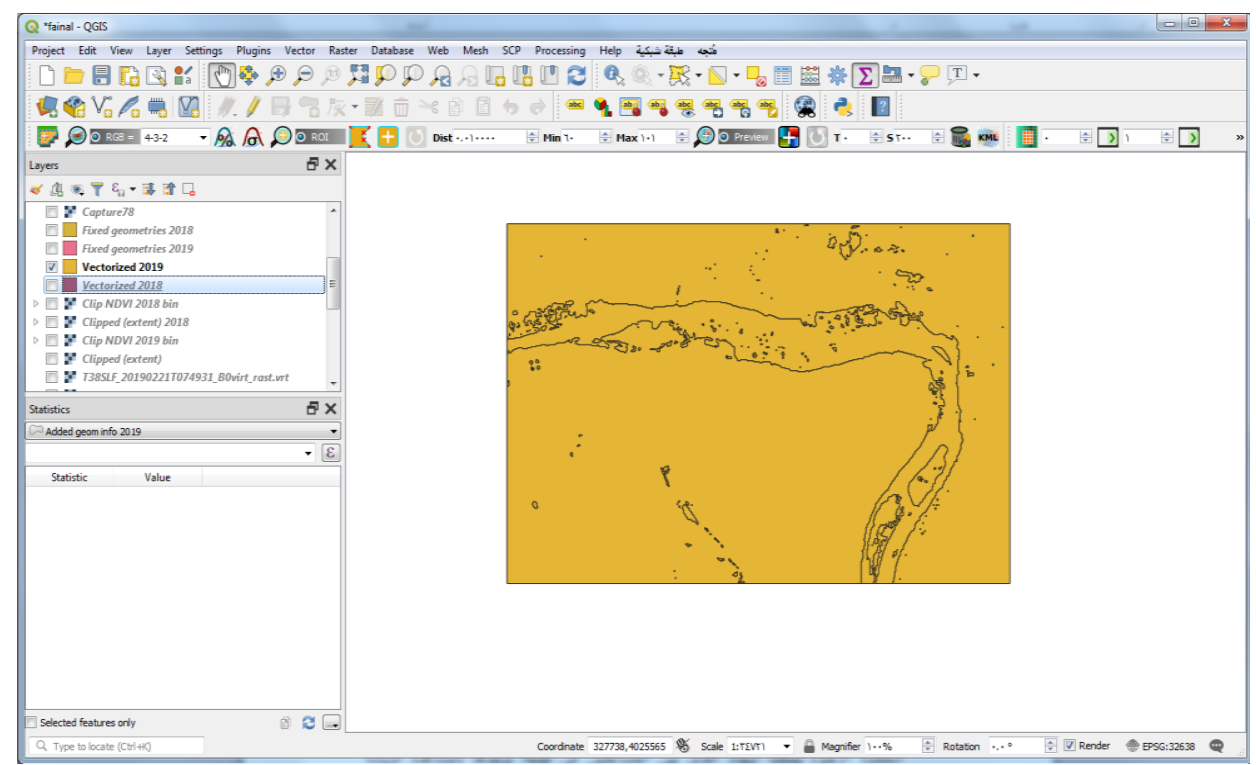

الثكل 8: تحويل المرئية من الصيغة الثبكية Raster الى الصيغة الثعاعية Vector.

الخطوة السادسة هي ايجاد المساحات باستخدام الايعاز (Added Geometry Attributes) الموجود في خانة معالجة الادوات (Processing tool box: Vector geometry) حيث سيتم ايجاد المساحة والمحيط لكل كائن موجود في المرئية. كما تم استخدام الفلتر لكي نحصل على مقطع النهر لوحده بدون الخفية (اي تصفية المرئية من المساحات الاصغر من مساحة النهر) وكما موضح في الثكل (9).

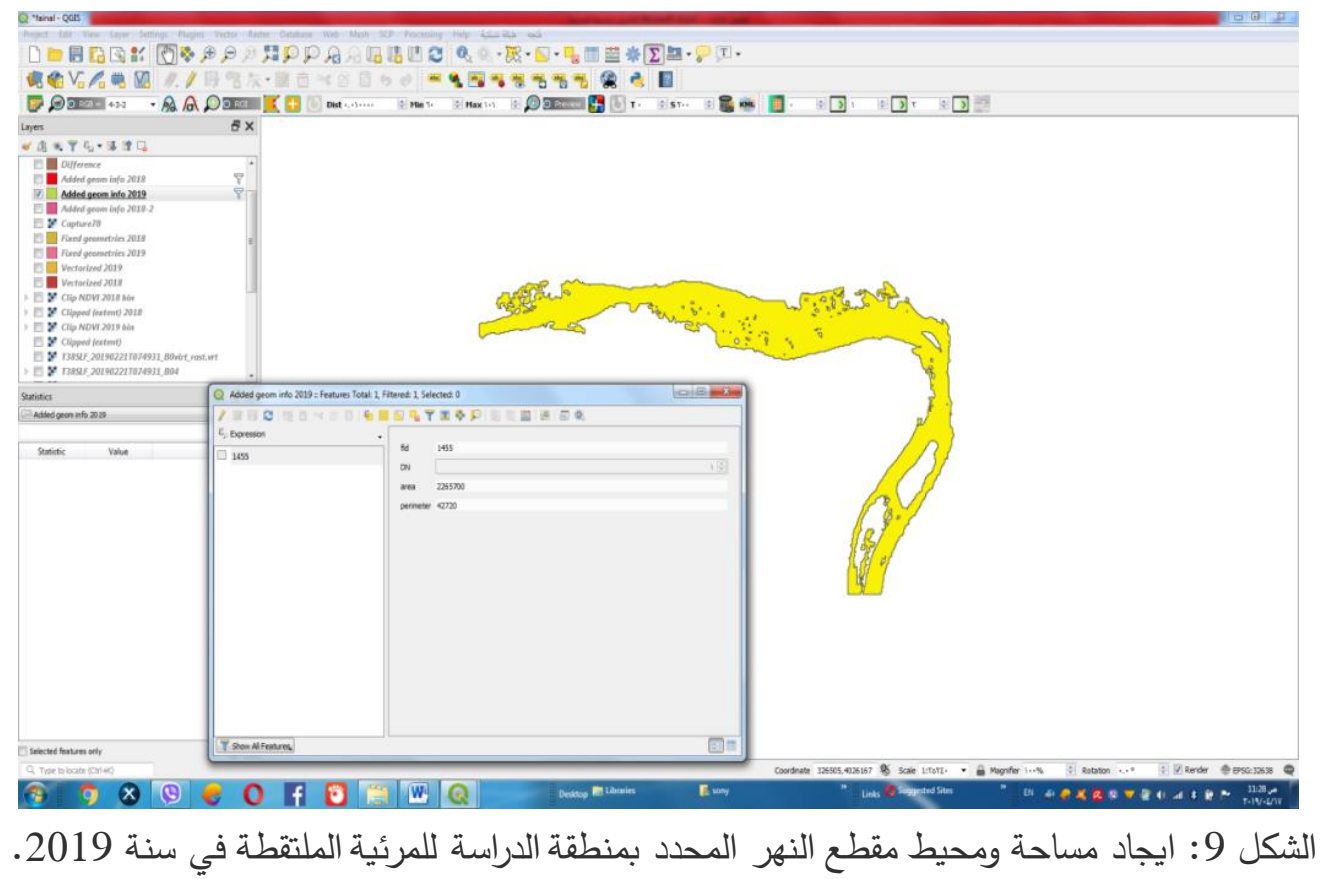

وفي الخطوة الاخيرة تم ايجاد الفرق بين المساحتين في طبقة لوحدها ومشاهدة الزيادة الحاصلة في سنة 2019 وكما مبين في الثكل (10)، حيث ان اللون الاحمر يبين مساحة مقطع النهر في السنة غير المطيرة 2018، والتي تم اجراء نفس خطوات المعالجة السابقة عليها، واللون الاصفر يبين مقدار الزيادة في السنة المطيرة 


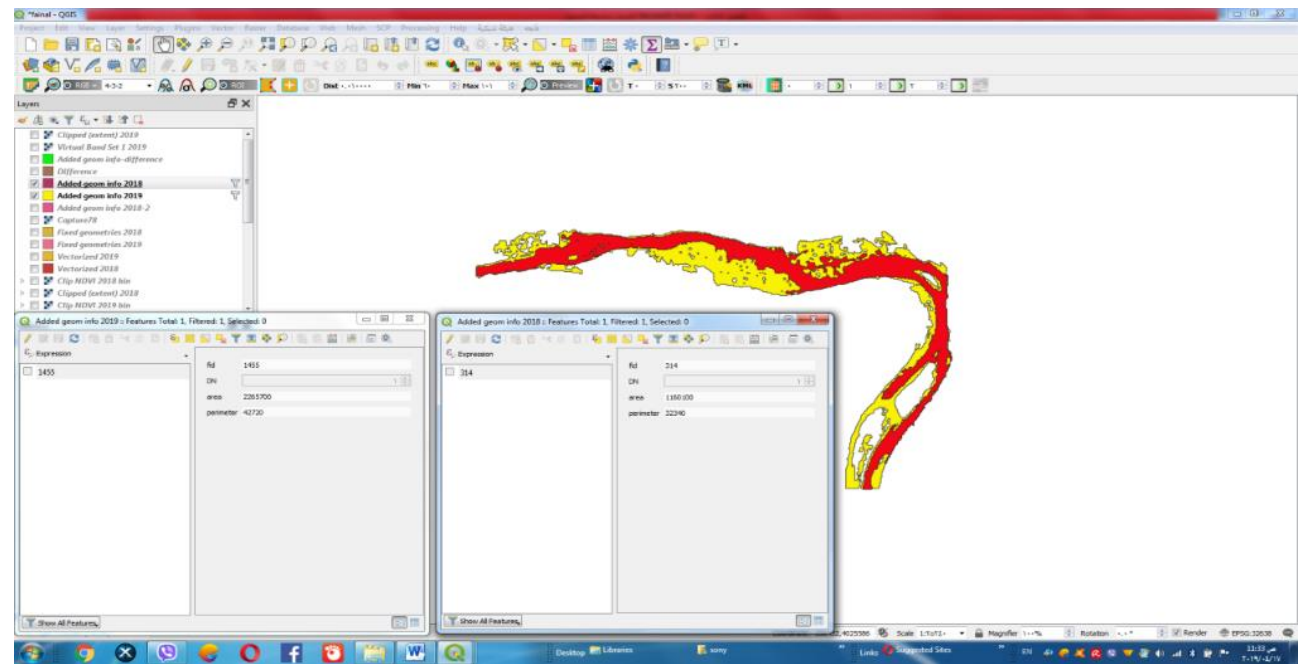

الثكل 10: ايجاد الفرق بين السنتين 2018 و2019 والذي يوضح المناطق الغارقة بالون الاصفر ضمن المنطقة.

\section{النتائتج}

بلغت مساحة مقطع النهر (1.160.100 كم²) في الثهر الثاني سنة 2018 في حين بلغت (265.700 كم²) في نفس الشهر لسنة 2019 اي ان الزيادة في مساحة مقطع النهر بلغت 1.105 كم² اي بزيادة أكثر من بن 50\% عن السنة السابقة كما موضح في الشكل (11). نستطيع بعد ذلك إنتاج خريطة سريعة في حالات الكوارث وكذلك حساب المساحات المغمورة والتغيرات الحاصلة على طول مجرى النهر وعلى كلا جانبي النهر.

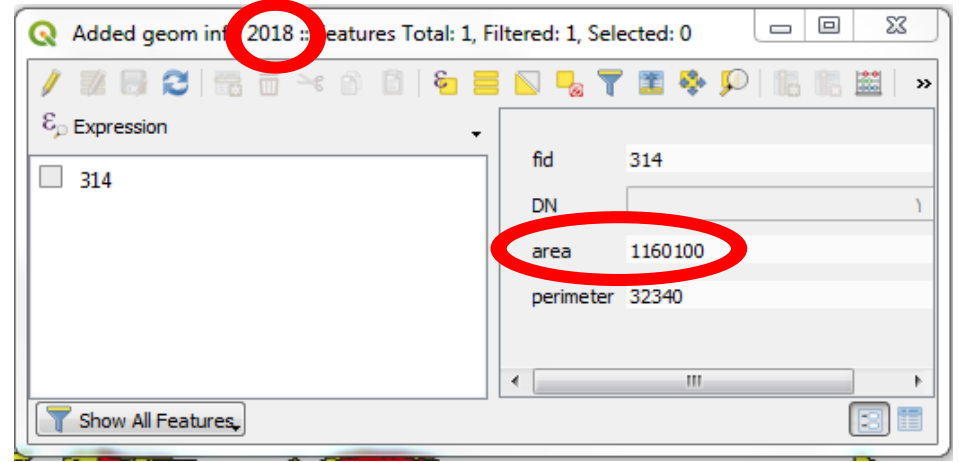

$-$

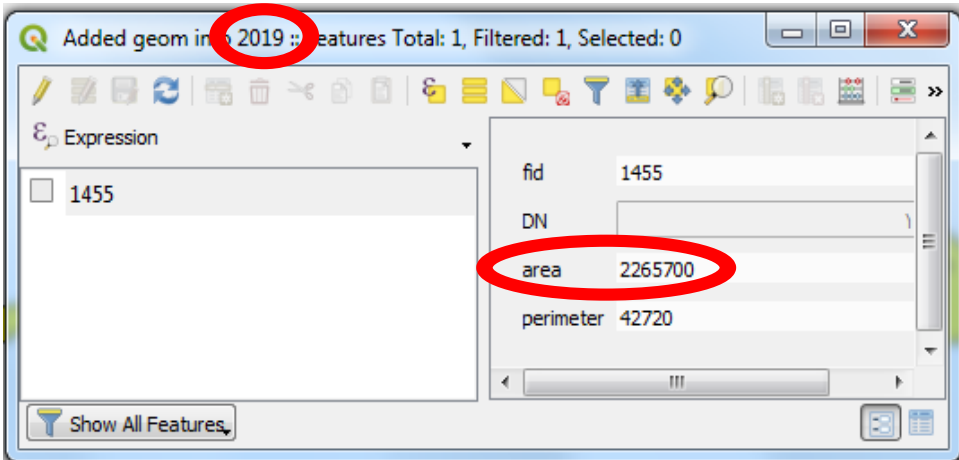

$-\varphi^{-}$

الشكل 11: شكل مكبر يوضح فرق الزيادة الحاصلة في المساحة السطحية للنهر في: --أ- السنة قليلة الامطار 2018، ب- السنة المطيرة 2019. وان الفرق بيزهما يمثل مساحة المناطق التي غرقت ضمن منطقة الدراسة. 


$$
\text { عبدالرحمن رمزي قبع و ايمن طلال الصائغ }
$$

يوضح الثكل (12) صور غرق المناطق الزراعية والتي تحولت الى مناطق سكنية عشوائية والمحاذية للنهر في منطقة الدراسة والملتقطة من قبل الباحثون نتيجة زيادة حجم حوض النهر وارتفاع منسوب المياه للموسم الثتوي المطري 2019 ضمن منطقة الدراسة، حيث تتوعت المنشآت المدنية المتأثرة على ضفة النهر بين مساكن وبين

مرافق ترفيهية.
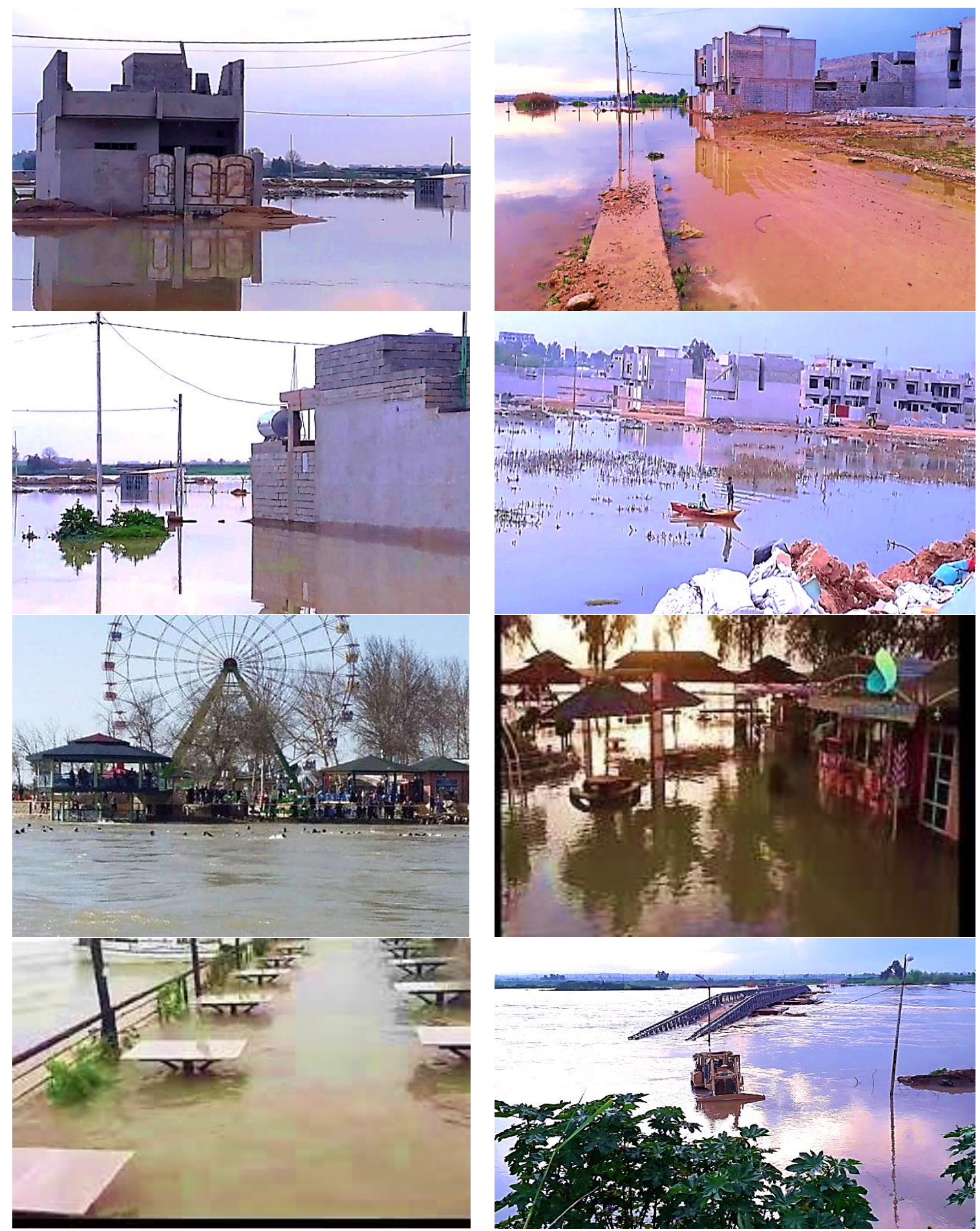

الثكل 12: توضح الصور اعلاه خطر موسم الفيضانات وتأثيره على المناطق السكنية والسياحية المستحدثة على اكتاف نهر دجلة وفي مقطع الدراسة والموثقة من قبل الباحثون داخل مدينة الموصل. 


\section{الاستنتاجات}

تكاملت في هذا البحث برامجيات نظم المعلومات الجغرافية وتقنيات التحس النائي ووسائل المعالجة الرقمية لبيان خطر السنوات المطيرة ولدرء خطر الفيضان والكوارث البشرية والمادية. حيث استخم برنامج نظم المعلومات الجغرافية الحديث QGIS لاستخلاص المعلومات ومراقبة التغيرات لمقطع من نهر دجلة ضمن مدينة الموصل في محافظة نينوى، كما تم استخدام مرئيتين فضائيتين لفترتين متعاقبتين بالزمن مصححة ومرجعة جغرافيا للقدر الاصطناعي الاوربي الجيد Sentinel2. بعد استقطاع منطقة الدراسة تم عمل تركيب لوني كاذب للمرئيتين باستخدام برنامج نظم المعلومات الجغرافية الجديد QGIS للحصول على أفضل تدرج لوني لتمييز المياه عن اليابسة باختيار الحزم الفضائية ذات الاطوال الموجية للطيف المرئي 4و32 وبدقة تمييز مكانية 10 م لكل بكسل. وقد أنجز تفير هذا التدرج اللوني للمساعدة في اعداد خرائط استخدامات الاراضي الواقعة على أكتاف نهر دجلة فضلا عن تحديد المناطق السكنية القريبة من مجرى النهر • تم عمل خريطة سريعة لمنطقة الفيضان بالقرب من النهر لما لهذه الخرائط من أهمية كبيرة لأعمال الانقاذ وتقدير الخسائر ووضع خطط لإدارة الكوارث والتعامل معها والاستفادة منها في الحد من ظاهرة الانتشار السكاني العشوائي بالقرب من أكتاف الأنهر. حيث بين البحث ان الزيادة في مساحة مقطع النهر في منطقة الدراسة بين السنة المطيرة والسنة القليلة الامطار بلغت 1.105 كم2 أي بزيادة تقريبية تجاوزت 50\%. كما بين البحث خطر بناء مساكن وأماكن ترفيهية في المناطق القريبة من أكتاف النهر ووجوب توجيه السلطات إلى استخدام التثنيات الحديثة ونظم المعلومات الجغرافية لمنع الاستحواذ العشوائي للأراضي الزراعية ومنع تحويلها من أراضي زراعية إلى أراضي سكنية لتجنب حوادث غرق هذه المناطق وتحذير مالكي الاماكن الترفيهية والكازينوهات المشيدة على أكتاف النهر الى خطر غرقها لقربها من ضفاف النهر. كما يوصي الباحثان بزيادة مساحة محرمات النهر الى نسبة تصل إلى أكثر من 50\% من مساحة اي مقطع سطحي للنهر داخل مدينة الموصل لتجنب كوارث الفيضانات كما حصل في حادثة غرق العبارة النهرية على ضفاف النهر في سنة 2019 التي راح ضحيتها أكثر من 150 شخص اغلبهم اطفال ونساء.

\section{المصادر العربية}

خطاب، محمد فوزي عمر ومصطفى، فاتن عزيز، 2009. قياس التغيرات الحاصلة في موقع مجرى نهر دجلة في

عامي 1987-2001 باستخدام معطيات التحس النائي. مجلة التربية والعلم، المجلد 16، العدد 2.

داؤد، جمعة محمد، 2014. مبادى علم نظم المعلومات الجغرافية. مكة المكرمة، المملكة العربية السعودية. شعبان، فادي عزالدين، 2019. الدليل التعليمي لبرنامج QGIS. كلية الهندسة المدنية، جامعة تشرين، الجمهورية السورية.

مقاييس، سجلات تصاريف نهر دجلة لمحطة الموصل القياسية صادرة عن مديرية ري نينوى ووزارة الموارد المائية، .2006 وزارة الموارد المائية، 2019. /http:/www.mowr.gov.iq. تم الولوج الى الموقع بتاريخ 2019/6/1. 


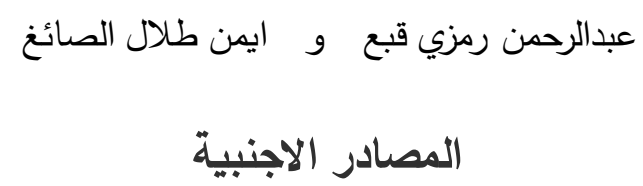

Belgiu, M. and Csillik O., 2018. Sentinel-2 cropland mapping using pixel-based and object-based time-weighted dynamic time warping analysis. Remote Sensing of Environment, Vol. 204, pp. 509-523.

Frampton W., J. Dash, G. Watmough and E. Milton, 2013. Evaluating the capabilities of Sentinel-2 for quantitative estimation of biophysical variables in vegetation. ISPRS Journal of Photogrammetry and Remote Sensing, V. 82, pp. 83-92.

Gatti A. and Galoppo A., 2018. Sentinel-2 Products Specification Document. REF: S2PDGS-TAS-DI-PSD, ISSUE: 14.5 .

https://sentinel.esa.int/documents/247904/685211/Sentinel-2-Products-SpecificationDocument

Jensen R., 2005. Introductory Digital Image Processing: A Remote Sensing Perspective, (4 Edition). Parson Education, 526 pages.

Lillesand M. Thomas. Thomas, Ralph W. Kiefer, and Jonathan W. Chipman, 2015. Remote Sensing and Image Interpretation. NJ: John Wiley \& Sons, Technology \& Engineering, 736 pages.

Zhang, Xi, George, B., Phil, G., Eve, H., and Lihong. Z, 2008. Integrating GIS and Remote Sensing with Urban Hydrology. ESRI Section User Conference Proceedings, San Diego. 